\title{
Hardy's Inequality for Functions of Several Complex Variables
}

(Ketidaksamaan Hardy untuk Fungsi Beberapa Pemboleh Ubah Kompleks)

\section{VANSAK SAM \& KAMTHORN CHAILUEK*}

\section{ABSTRACT}

We obtain a generalization of Hardy's inequality for functions in the Hardy space $H^{1}\left(\mathbb{B}_{d}\right)$, where $\mathbb{B}_{d}$ is the unit ball $\left\{z=\left.\left(z_{1}, \ldots, z_{d}\right) \in \mathbb{C}^{d}\left|\Sigma_{i=1}^{d}\right| z_{i}\right|^{2}<1\right\}$. In particular, we construct a function $\phi$ on the set of $d$-dimensional multi-indices $\left\{n=\left(n_{1}, \ldots, n_{d}\right) \mid n_{i} \in \mathbb{N} \cup\{0\}\right\}$ and prove that if $f(z)=\sum a_{n} z^{n}$ is a function in $H^{1}\left(\mathbb{B}_{d}\right)$, then $\sum_{|n|=0}^{\infty} \frac{\left|a_{n}\right|}{\phi(n)+1} \leq \pi|| f \|_{1}$. Moreover, our proof shows that this inequality is also valid for functions in Hardy space on the polydisk $H^{1}\left(\mathbb{B}^{d}\right)$.

Keywords: Hardy's inequality; Hardy space and Hilbert's inequality

\section{ABSTRAK}

Kami memperoleh generalisasi ketidakseimbangan Hardy's untuk fungsi dalam ruang Hardy $H^{1}\left(\mathbb{B}_{d}\right)$, dengan $\mathbb{B}_{d}$ adalah unit bola $\left\{z=\left.\left(z_{1}, \ldots, z_{d}\right) \in \mathbb{C}^{d}\left|\Sigma_{i=1}^{d}\right| z_{i}\right|^{2}<1\right\}$. Secara khususnya, kami membina fungsi $\phi$ pada set indeks pelbagai $d$-dimensi $\left\{n=\left(n_{1}, \ldots, n_{d}\right) \mid n_{i} \in \mathbb{N} \cup\{0\}\right\}$ dan membuktikan bahawa jika $f(z)=\Sigma a_{n} z^{n}$ adalah fungsi di $H^{1}\left(\mathbb{B}_{d}\right)$, kemudian $\sum_{|n|=0}^{\infty} \frac{\left|a_{n}\right|}{\phi(n)+1} \leq \pi\|f\|_{1}$. Selain itu, bukti kami menunjukkan bahawa ketidakseimbangan ini juga adalah sah untuk fungsi dalam ruang Hardy ke atas polidisk $H^{1}\left(\mathbb{B}^{d}\right)$.

Kata kunci: Ketidaksamaan Hardy; Ruang Hardy dan ketidaksamaan Hilbert

\section{INTRODUCTION}

For $z=\left(z_{1}, \ldots, z_{d}\right)$ in the $d$-dimensional complex Euclidean space $\mathbb{C}^{d}$, we define $\|z\|^{2}=\sum_{i=1}^{d}\left|z_{i}\right|^{2}$. Let $\mathbb{B}_{d}$ denote the open unit ball containing $z \in \mathbb{C}^{d}$ such that $\|z\|^{2}<1$. A function $f: \mathbb{B}_{d} \rightarrow \mathbb{C}$ is holomorphic if for each $i=1, \ldots, d$ and each fixed $z_{1}, \ldots, z_{i-1}, z_{i+1}, \ldots, z_{d}$, the function $f_{i}: \xi \mapsto f\left(z_{1}\right.$, $\left.\ldots, z_{i-1}, \xi, z_{i+1}, \ldots, z_{d}\right)$ is holomorphic as a function of one variable. For $0<p<\infty$, the Hardy space $H^{p}\left(\mathbb{B}_{d}\right)$ consists of all holomorphic functions $f$ defined $\mathbb{B}_{d}$ on satisfying

$$
\|f\|_{p}^{p}=\sup _{0<r<1} \int_{\mathrm{S}_{d}}|f(r \xi)|^{p} d \sigma(\xi)<\infty
$$

where $\mathbb{S}_{d}$ is the boundary of $\mathbb{B}_{d}$ and $d \sigma$ is the normalized surface measure. Note that one can also define Hardy space of functions defined on the polydisk $\mathbb{B}^{d}=\mathbb{B} \times \ldots \times \mathbb{B}$ as the space of holomorphic functions $f$ satisfying:

$$
\sup _{0<r<1} \int_{[0,2 \pi]^{d}} \mid f\left(\left.r e^{i \theta}\right|^{p} \frac{d \theta}{(2 \pi)^{d}}<\infty,\right.
$$

where $e^{i \theta}=\left(e^{i \theta_{1}}, \ldots, e^{i \theta_{d}}\right)$ and $d \theta=d \theta_{1} \ldots d \theta_{d}$.
For the case $p=1$, Hardy's inequality for functions of one variable defined on the unit ball in $\mathbb{C}$ is well-known. It states that if $f(z)=\sum_{n=0}^{\infty} a_{n} z^{n} \in H^{1}$, then

$$
\sum_{n=0}^{\infty} \frac{\left|a_{n}\right|}{n+1} \leq \pi\|f\|_{1}
$$

see Duren (1970).

There are some connections between Hardy's inequality and inequalities in other Hilbert spaces. For example, Zhu (2004) translated this Hardy's inequality to the inequality $\sum_{n=0}^{\infty} \frac{\Gamma(\alpha+2) \Gamma\left(\frac{\pi}{2}+1\right)}{(n+1) \Gamma\left(\frac{\pi}{2}+\alpha+2\right)}\left|a_{n}\right| \leq \pi \int_{\mathbb{B}}|f(z)| d A_{\alpha}(z)$ for functions $f=\sum_{n=1}^{\infty} a_{n} z^{n}$ in the Bergman space $A_{\alpha}^{1}$.

Sometimes, Hardy's inequality appears in an integral form. In Sababheh (2008a, 2008b), the author proved Hardy-type inequalities concerning the integral of the Fourier transform $\hat{f}$ of a function $f$ with certain properties. 
That is, for $f \in L^{1}(\mathbb{R})$ with $\int^{x} f(t) d t \in L^{1}(\mathbb{R}), \hat{f}(\xi)=0$ when $\xi<0$ and $\alpha>2$, the inequality $\int_{0}^{\infty} \frac{|\hat{f}(\xi)|^{\alpha}}{\xi} d \xi \leq\left. 2 \pi|| f\right|^{\alpha}$ holds.

There is also a generalization on the multiplier $\frac{1}{n+1}$ in the summation of Hardy's inequality. Paulsen and Singh (2015) replaced the term $\frac{1}{n+1}$ in $\sum_{n=0}^{\infty} \frac{\left|a_{n}\right|}{n+1} \leq \pi\|f\|_{1}$ by a larger class of sequences. They proved that there is a constant $A$ therefore if $\left(c_{n}\right)$ is a sequence in some specific sequence space, then for any $f$ in Hardy space $H^{1}$, the inequality holds $\sum_{n=0}^{\infty}\left|c_{n} a_{n}\right| \leq A|| c_{n}\|\mid f\|_{1}$. This generalization is for a function $f$ of one variable.

To generalize Hardy's inequality to functions $f$ of several complex variables, we need to concern whether our functions are defined on the unit ball $\mathbb{B}_{d}$ or the polydisk $\mathbb{B}^{d}$. Basically, we cannot apply iterated integrals ( $d$-times) to a function in $H^{p}\left(\mathbb{B}_{d}\right)$ as we usually do to functions in $H^{p}\left(\mathbb{B}^{d}\right)$.

In this paper, we will show that we can adjust to the proof in Duren (1970) to obtain Hardy's inequality that is valid for functions in either $H^{p}\left(\mathbb{B}_{d}\right)$ or $H^{p}\left(\mathbb{B}^{d}\right)$. A difficulty in the case of functions of several complex variables is that a holomorphic function $f$ is represented by $f(z)=\Sigma a_{n} z^{n}$ where $n=\left(n_{1}, \ldots, n_{d}\right)$ is a multi-index. However, we will show that the set of multi-indices can be totally ordered in the way which enables us to prove a generalized Hardy's inequality.

\section{MAIN THEOREMS}

For multi-indices $n=\left(n_{1}, \ldots, n_{d}\right)$ and $m=\left(m_{1}, \ldots, m_{d}\right)$ in $\mathbb{N}_{0}^{d}$ where $\mathbb{N}_{0}=\{0,1,2,3, \ldots\}$, we define $|n|=\sum_{i=1}^{d} n_{i}$, $n !=n_{1} ! \ldots n_{d} !$ and $n \pm m=\left(n_{1} \pm m_{1}, \ldots, n_{d} \pm m_{d}\right)$. For $z=\left(z_{1}, \ldots, z_{d}\right) \in \mathbb{C}^{d}$, we also define $z^{n}=z_{1}^{n_{d}} \ldots z_{d}^{n_{d}}$. With this notation and for a given $k \in \mathbb{N}_{0}$, there are $\frac{(k+d-1) !}{k !(d-1) !}$ terms of $a_{n} z^{n}$ when $|n|=k$.

First we consider a lemma by Peter Duren which defines a bilinear form on vectors $x=\left(x_{n}\right)$ and $y=\left(y_{n}\right)$ in $\mathbb{C}^{N}$ and prove that it is bounded. We then generalize this result to the case where $n$ are multi-indices. This result plays an important role in proving the Hilbert's inequality in Lemma 3 and Hardy's inequality in Theorem 2.

Lemma 1. Let $\psi \in L^{\infty}([0,2 \pi])$ and $\lambda_{n}=\frac{1}{2 \pi} \int_{0}^{2 \pi} e^{-i n t} \psi(t) d t$, $n=0,1,2, \ldots$. Let $x=\left(x_{1}, \ldots, x_{N}\right)$ and $y=\left(y_{1}, \ldots, y_{N}\right)$ be vectors in $\mathbb{C}^{N}$. Define

$$
A_{N}(x, y)=\sum_{n, m=0}^{N} \lambda_{n+m} x_{n} y_{m} .
$$

Then

$$
\left|A_{N}(x, y)\right| \leq\|\psi\|_{\infty}\|\| x\|\| y \| .
$$

Proof. This proof is due to Duren (1970),

$$
\begin{aligned}
& \left|A_{N}(x, x)\right| \\
& =\left|\sum_{n, m=0}^{N} \lambda_{n+m} x_{n} x_{m}\right| \\
& =\left|\frac{1}{2 \pi} \int_{0}^{2 \pi} \sum_{n, m=0}^{N} e^{-i n t} x_{n} \cdot e^{-i m t} x_{m} \psi(t) d t\right| \\
& =\mid \frac{1}{2 \pi} \int_{0}^{2 \pi}\left(\left.\sum_{n=0}^{N} e^{-i n t} x_{n}\right|^{2} \psi(t) d t \mid\right. \\
& \quad \leq \frac{1}{2 \pi} \int_{0}^{2 \pi}\left|\sum_{n=0}^{N} e^{-i n t} x_{n}\right|^{2} \mid \psi \psi \|_{\infty} d t \\
& =\frac{|| \psi \|_{\infty}}{2 \pi} \int_{0}^{2 \pi}\left|\sum_{n=0}^{N} e^{-i n t} x_{n}\right|^{2} d t .
\end{aligned}
$$

Now, we obtain,

$$
\left|\sum_{n=0}^{N} e^{-i n t} x_{n}\right|^{2}=\left(\sum_{n=0}^{N} e^{-i n t} x_{n}\right)\left(\sum_{n=0}^{N} e^{-i \text { int }} \bar{x}_{n}\right)=\sum_{n=0}^{N}\left|x_{n}\right|^{2}+\sum_{n \neq m} e^{i(m-n) t} x_{n} \bar{x}_{m} .
$$

Note that,

$$
\int_{0}^{2 \pi} e^{i k t} d t=0
$$

if $k \neq 0$. Therefore,

$$
\left|A_{N}(x, x)\right| \leq\left.\frac{\|\psi\|_{\infty}}{2 \pi} \int_{0}^{2 \pi} \sum_{n=0}^{N} e^{-i n t} x_{n}\right|^{2} d t=\frac{\|\psi\|_{\infty}}{2 \pi} 2 \pi\|x\|^{2}=\|\psi\|_{\infty}\|x\|^{2} .
$$

This bilinear form also satisfies the polarization identity

$$
A_{N}(x, y)=\frac{1}{4} A_{N}(x+y, x+y)-\frac{1}{4} A_{N}(x-y, x-y) .
$$

Then by the parallelogram law,

$$
\|x+y\|^{2}+\|x-y\|^{2}=2\|x\|^{2}+2\|y\|^{2},
$$

we obtain

$$
\begin{aligned}
& \left|A_{N}(x, y)\right|=\frac{1}{4}\left|A_{N}(x+y, x+y)-A_{N}(x-y, x-y)\right| \\
& \leq \frac{1}{4}\left(\left|A_{N}(x+y, x+y)\right|+\left|A_{N}(x-y, x-y)\right|\right) \\
& =\frac{1}{4} \|\left(\psi\left\|_{\infty}\left(\|x+y\|^{2}+\|x-y\|^{2}\right)=\frac{1}{2}\right\| y \|_{\infty}\left(\|x\|^{2}+\|y\|^{2}\right) .\right.
\end{aligned}
$$

We can see that when $\|x\|=\|y\|=1,\left|A_{N}(x, y)\right| \leq\|\psi\|_{\infty}$.

Hence $\left|A_{N}(x, y)\right| \leq\|\psi\|_{\infty}\|x\|\|y\|$. 
To generalize this inequality to the case of multiindices $n=\left(n_{1}, \ldots, n_{d}\right)$, we need a new formula for $\lambda_{n}$. Then, if we can find an upper bound of $\left|A_{N}(x, x)\right|$ in terms of $\|x\|$, we automatically obtain an upper bound of $\left|A_{N}(x, y)\right|$. The reason is that the rest of this proof depends only on properties of the norm.

Now, let $x=\left(x_{n}\right)$ be a vector where $n$ is a multi-index with $|n|=0,1, \ldots, N$. For example, if $N=3$ and $d=2$, then a vector $\left(x_{n}\right)$ could be $\left(x_{00}, x_{01}, x_{10}, x_{11}, x_{20}, x_{12}, x_{21}, x_{30}, x_{03}\right)$. Note that we can consider $\left(x_{n}\right)$ when $0 \leq|n| \leq N$ as a finite sequence with $\sum_{k=0}^{N} \frac{(k+d-1) !}{k !(d-1) !}$ terms. We also define $\|x\|$ to be $\sqrt{\sum_{|n|}^{N}\left|x_{n}\right|^{2}}$.

Now, when $n$ is a multi-index, the term $e^{-i n t}$ in the formula for $\lambda_{n}$ is invalid. For the first try, one may replace $n$ by $|n|$ and let $\lambda_{n}=\frac{1}{2 \pi} \int_{0}^{2 \pi} e^{-i n \mid t} \psi(t) d t$. Unfortunately, the map $||:. n \mapsto|n|$ is not injective. There exist multi-indices $r, s$ such that $r \neq s$ but $|r|=|s|$, for example

$$
|(1,0,0, \ldots, 0)|=|(0,1, \ldots, 0)| .
$$

Therefore,

$$
\frac{1}{2 \pi} \int_{0}^{2 \pi} \sum_{n \neq m}^{i(|n|-m|n| t} x_{n} \overline{x_{m}} d t \neq 0
$$

and hence

$$
\frac{1}{2 \pi} \int_{0}^{2 \pi}\left|\sum_{n=0}^{N} e^{-i n t} x_{n}\right|^{2} d t \neq \|\left. x\right|^{2}
$$

Thus we will not obtain an analogue of inequality (2). However, it suggests that if we have an injective function $\phi(n)$ on the set of multi-indices and let

$$
\lambda_{n}=\frac{1}{2 \pi} \int_{0}^{2 \pi} e^{-i \phi(n) t} \psi(t) d t,
$$

then the proof of Lemma 1 will also be valid for the case where $n$ is a multi-index. This will lead to Lemma 2 below.

Before we state Lemma 2, let us discuss the existence of $\phi$. We know from the Zermelo's well-ordering theorem that every set can be well-ordered (and hence totally ordered) which implies the existence of an injective function $\phi$ from any set to the set $\mathbb{N}$. However, the proof of the Zermelo's well-ordering theorem is non-constructive. Below, we s' give an explicit construction of an injective function $\phi$ from the set of all multi-indices to the set $\mathbb{N}$.

The following Lemma 2 and Lemma 3 hold for an arbitrary injective function $\phi$. However Theorem 1 requires that $\phi$ has to be independent of the order $N$ of a multi-index $n$. This is because, in the proof of Theorem 1, we find an upper bound of the summation $\sum_{\mid n=0}^{N} \lambda_{n}\left|a_{n}\right|$, in the following
Inequality (3). Then we take $N \rightarrow \infty$ to obtain an upper bound of $\sum_{|n|=0}^{\infty} \lambda_{n}\left|a_{n}\right|$. This strategy suggests that $\lambda_{n}$ must be independent of $N$.

We first look for a function $\phi$ defined on the set of multi-indices which is independent of $N$. Consider the relation $\leq$ for multi-index notation. We say that $n \leq m$ if $n$ $\leq m_{i}$ for all $i$. This relation is merely partially ordered and, for example, we cannot compare $(1,0,1)$ and $(0,1,0)$. Now, for $n \neq m$, we denote $n \prec m$ if,

1. $|n|<|m|$ or

2. $|n|=|m|$ with

$$
n_{1} n_{2} \ldots n_{d|n|+1}<m_{1} m_{2} \ldots m_{d|n|+1}
$$

where $n_{1} n_{2} \ldots n_{d|n|+1}$ is the representation of a number in base $|n|+1$.

Precisely,

$$
\left.n_{1} n_{2} \ldots n_{d|n|+1}=\sum_{k=1}^{d} n_{k}|n|+1\right)^{d-k} .
$$

Now, the relation $\prec$ is totally ordered. Since the relation $\prec$ is totally ordered, we can construct an injective function $\phi$ defined according $\prec$ to as follows.

For example, when $d=3$, we have $(0,0,0) \prec(0,0,1)$ $\prec(0,1,0) \prec(1,0,0) \prec(0,0,2) \prec(0,1,1) \prec(0,2,0) \prec$ $(1,0,1) \prec(1,1,0) \prec(2,0,0) \prec(0,0,3) \prec \ldots$.

It is easy to see that we arrange the multi-indices $n$ according to their order $|n|$. Then, among multi-indices with the same order, we arrange them according to their values in base $|n|+1$, each of which is a unique representation.

Then we define $\phi(n)$ according to the arrangement of $n$ via the relation $\prec$. As in this example, we obtain $\phi((0$, $0,0))=0, \phi((0,0,1))=1, \phi((0,1,0)=2, \phi((1,0,0))=3$, $\phi((0,0,2)=4, \ldots$ We note that $\phi$ is injective. When $d=$ 1 , we also have $\phi(n)=n$. We now generalize Lemma 1 to the following lemma for vectors $\left(x_{n}\right)$ and $\left(y_{n}\right)$ where $n$ is a multi-index.

Lemma 2. Let $\psi \in L^{\infty}([0,2 \pi]), N \in \mathbb{N}, N=\left\{n=\left(n_{1}\right.\right.$, $\left.\ldots, n_{d}\right): 0 \leq|n| \leq N$, and $\phi$ be an injective function from $N$ to $\mathbb{N}$. Let

$$
\lambda_{n}=\frac{1}{2 \pi} \int_{0}^{2 \pi} e^{-i \phi(n) t} \psi(t) d t,
$$

$n=\left(n_{1}, \ldots, n_{d}\right) \in \mathbb{N}_{0}^{d}$ and $A_{N}(x, y)=\sum_{|n|,|m|=0}^{N} \lambda_{n+m} x_{n} y_{m}$.Then $\left|A_{N}(x, y)\right| \leq\|\psi\|_{\infty}\|x\| y \|$.

Proof. The proof of this lemma is analogous to that of Lemma 1.

In the previous lemmas, the function $\psi$ is an arbitrary function in $L^{\infty}([0,2 \pi])$ and Lemma 2 is valid for any injective function $\phi$. Next, in Lemma 3 (and also later 
in Theorem 2), we will choose a specific function $\psi$, i.e. we will use $\psi(t)=i e^{-i t}(\pi-t)$. This will fix $\|\psi\|_{\infty}$ and thus a constant in the equality. With this specific choice of $\psi$ together with an injective function $\phi$, we define $\lambda_{n+m}$ and compute $\left|\lambda_{n+m}\right|$, as well as $\|\psi\|_{\infty}$. Applying this result to the inequality in Lemma 2, we obtain another version of Hilbert's inequality.

\section{Lemma 3. Let $N \in \mathbb{N}$,}

$\mathcal{N}=\left\{n=\left(n_{1}, \ldots, n_{d}\right): 0 \leq|n| \leq N\right\}$, and $\phi$ be an injective function from $\mathcal{N}$ to $\mathbb{N}$. Then

$$
\left|\sum_{|n|,|m|=0}^{N} \frac{x_{n} y_{m}}{\phi(n+m)+1}\right| \leq \pi|| x \mid\|y\| .
$$

Proof. Choose $\psi(t)=i e^{-i t}(\pi-t)$. By Lemma 2, we have $\lambda_{n+m}$

$$
\begin{aligned}
& =\frac{1}{2 \pi} \int_{0}^{2 \pi} e^{-i \phi(n+m) t} i e^{-i t}(\pi-t) d t \\
& =\frac{1}{2 \pi} i \int_{0}^{2 \pi} e^{-i(\phi(n+m)+1) t}(\pi-t) d t \\
& =\frac{1}{2 \pi} i\left(\pi \int_{0}^{2 \pi} e^{-i(\phi(n+m)+1) t} d t-\int_{0}^{2 \pi} e^{-i(\phi(n+m)+1) t} t d t\right) .
\end{aligned}
$$

By the Euler formula

$$
e^{i x}=\cos x+i \sin x
$$

the first integral can be eliminated and the second integral can be decomposed as

$$
\int_{0}^{2 \pi} t \cos [(\phi(n+m)+1) t] d t+i \int_{0}^{2 \pi} t \sin [(\phi(n+m)+1) t] d t .
$$

Using integration by parts, we also obtain

$$
\int_{0}^{2 \pi} t \cos [(\phi(n+m)+1) t] d t=0 .
$$

However,

$$
\int_{0}^{2 \pi} t \sin [(\phi(n+m)+1) t] d t=-\frac{2 \pi}{\phi(n+m)+1} .
$$

Therefore, $\left|\lambda_{n+m}\right|=\frac{1}{\phi(n+m)+1}$. Consider $|\psi(t)|=\left|i e^{-i t}(\pi-t)\right|=$ $\mid \pi-t) \mid \leq \pi$, for all $t$. Therefore, $\|\left.\psi\right|_{\infty} \leq \pi$. Then, by Lemma 2,

$$
\left|\sum_{|n|,|m|=0}^{N} \frac{x_{n} y_{m}}{\phi(n+m)+1}\right| \leq \pi\|x\|\|y\|
$$

Now we shall consider a function $f \in H^{1}\left(\mathbb{B}_{d}\right)$. Suppose that the Taylor expansion of $f$ is of the form $f(z)=\sum a_{n} z^{n}$. Then, by orthogonality of $\left\{z^{n}\right\}$ as functions in $H^{2}$, we can compute the norm of $f$ in terms of the sum of the square of the Taylor coefficients $\Sigma\left|a_{n}\right|^{2}$. The next theorem shows a relation between a weighted sum of coefficients in the Taylor expansion of $f \in H^{1}$ and the norm $\|f\|_{1}$.

\section{Theorem 1. Let}

$$
f(z)=\sum_{|n|=0}^{\infty} a_{n} z^{n} \in H^{1} \text { and } \lambda_{n} \geq 0 \text {. Then } \sum_{|n|=0}^{\infty} \lambda_{n}\left|a_{n}\right| \leq\|\psi\|_{\infty}|| f \|_{1} .
$$

Proof. Since $f \in H^{1}$, there exist $g$ and $h$ in the same $H^{2}$ such that $f=g h$ and $\|g\|_{2}^{2}=\|h\|_{2}^{2}=\|f\|_{1}$. We can also write $g$ and $h$ as $g(z)=\sum_{|n|=0}^{\infty} b_{n} z^{n}$ and $h(z)=\sum_{|n|=0}^{\infty} c_{n} z^{n}$. Consider,

$$
f(z)=\sum a_{n} z^{n}=\left(\sum b_{n} z^{n}\right)\left(\sum c_{n} z^{n}\right) .
$$

For the case $d=1$, it is easy to verify that $a_{n}=\sum_{|n|=0}^{\infty}$ $b_{k} c_{n-k}$. For $d \geq 1$, the product $b_{k} z^{k} c_{s} z^{s}$ is of the form

$$
b_{k_{1}, \ldots, k_{d}} c_{s_{1}, \ldots, s_{d}} z_{1}^{k_{1}+s_{1}} \ldots z_{d}^{k_{d}+s_{d}} .
$$

Therefore, to obtain

$$
a_{n} z^{n}=a_{n_{1}, \ldots, n_{d}} z_{1}^{n_{1}} \ldots z_{d}^{n_{d}}
$$

we need all possible choices of $k=\left(k_{1}, \ldots, k_{d}\right)$ and $s=\left(s_{1}\right.$, $\ldots, s_{d}$ ) such that $s=n-k$, which is the same as in the case $d=1$. Therefore, we also obtain $a_{n}=\Sigma_{0 \leq k \leq n} b_{k} c_{n-k}$. However, we should note that, for the case $d=1$, there are $n+1$ terms in $a_{n}=\sum_{k=0} b_{k} c_{n-k}$ whereas there are $\left(n_{1}+1\right) .\left(n_{2}+1\right) \ldots$ $\left(n_{d}+1\right)$ terms in $a_{n}=\Sigma_{0 \leq k \leq n} b_{k} c_{n-k}$ for the case $d \geq 1$. Then, by the triangle inequality, we have

$$
\begin{aligned}
& \sum_{|n|=0}^{N} \lambda_{n}\left|a_{n}\right|=\sum_{\mid n=0}^{N} \lambda_{n}\left|\sum_{0 \leq k \leq n} b_{k} c_{n-k}\right| \\
& \leq \sum_{\mid n=0}^{N} \lambda_{n} \sum_{0 \leq k \leq n}\left|b_{k}\right|\left|c_{n-k}\right|
\end{aligned}
$$

The summation $\Sigma_{0 \leq k \leq n}\left|b_{k}\right|\left|C_{n-k}\right|$ depends on $n$. Therefore,

$$
\sum_{|n|=0}^{N} \lambda_{n} \sum_{0 \leq k \leq n}\left|b_{k}\right|\left|c_{n-k}\right| \leq \sum_{|k||m|=0}^{N} \lambda_{k+m}\left|b_{k}\right|\left|c_{m}\right| \leq\left\|\psi \left|\left\|_{\infty}|| g\left|\|_{2}\right||h|_{2} .\right.\right.\right.
$$

The last inequality is a consequence of Lemma 2. Since $\|g\|_{2}\|h\|_{2}=\|f\|_{1}$, we obtain

$$
\sum_{\mid n=0}^{N} \lambda_{n}\left|a_{n}\right| \leq\|\psi\|\left\|_{\infty}|| f\right\|_{1}
$$

for any $N$. By letting $N \rightarrow \infty$, we obtain $\sum_{\mid n=0}^{\infty} \lambda_{n}\left|a_{n}\right| \leq|| \psi\left|\left\|_{\infty}|| f\right\|_{1}\right.$.

Next, we will show that the Hardy's inequality for functions of several complex variables can be easily proved by using Theorem 1 together with the function $\psi$ defined in Lemma 3. 
Theorem 2. If $f(z)=\sum_{|n|=0}^{\infty} a_{n} z^{n} \in H^{1}$ and $\phi$ is an injective function from the set of multi-indices to the set $\mathbb{N}$, then

$$
\sum_{|n|=0}^{\infty} \frac{\left|a_{n}\right|}{\phi(n)+1} \leq \pi\|f\|_{1},
$$

Proof. Let $f$ be any function in $H^{1}$ and $\psi(t)=i e^{-i t}(\pi-t)$ for $0 \leq t \leq 2 \pi$. Then $\|\psi\|_{\infty} \leq \pi$. As in the proof of Lemma 3 , we obtain $\left|\lambda_{n}\right|=\frac{1}{\phi(n)+1}$. Then Inequality (4) follows from Theorem 1. We shall also call Inequality (4) Hardy's inequality.

\section{DISCUSSION}

Our Hardy's inequality (4) comes directly from Inequality (3) in Theorem 1. With our specific choice of function $\psi$, we have $\|\psi\|_{\infty} \leq \pi$ and $\left|\lambda_{n}\right|=\frac{1}{\phi(n)+1}$. The latter holds for any injective function $\phi$ which is independent of $f$. The proof of Theorem 1 involves only the coefficients of the Taylor expansion of $f$, regardless of where $f$ is defined. Therefore, Hardy's inequality (Inequality (4)) holds for all functions $f$ in Hardy space $H^{1}\left(\mathbb{B}_{d}\right)$ as well as functions $f$ in $H^{1}\left(\mathbb{B}^{d}\right)$.

Let us note that Lemmas 2 and 3 are true for any injective function $\phi$ defined on the set of multi-indices $n$ when $0 \leq|n| \leq N$ and they do not require that $\phi$ has to be $N$-independent. For example, let us consider a function $\Phi$ defined by

$$
\left.\Phi\left(n_{1}, n_{2}, \ldots, n_{d}\right)\right)=n_{1} n_{2} \ldots n_{d_{N+1}},
$$

which is also injective but less complicated than the function $\varphi$ we constructed earlier. The proof of Lemma 3 is also true for this function $\Phi$. However, we cannot use this $\Phi$ in Theorem 1 because the formula for $\Phi$ depends on $N$ which will cause a problem when we take $N \rightarrow \infty$.

The proof of Theorem 2 is valid for any injective function from the set of multi-indices to the set $\mathbb{N}$. Our specific example $\phi$ (constructed before Lemma 2) has a property that $\phi(n)=n$ when $d=1$. Thus Inequality (4) reduces to Hardy's inequality $\sum_{n=0}^{\infty} \frac{\left|a_{n}\right|}{n+1}$ when $d=1$. Suppose that $\phi$ is another injective function such that when $d=1$, the value $\phi(n)$ is not necessarily equal to $n$. Then, Inequality (4) will yield another version of Hardy's inequality for $d=1$, where the denominators $n+1$ of the summation $\sum_{n=0}^{\infty} \frac{\left|a_{n}\right|}{n+1}$ in the standard Hardy's inequality will be replaced by a sequence of distinct integers greater than 1. Therefore, not only that Inequality (4) generalizes the standard Hardy's inequality to the case $d>1$, it also gives a generalization in the case $d=1$.

\section{REFERENCES}

Duren, P. 1970. Theory of Spaces. New York: Academic Press. Paulsen, V.I. \& Singh, D. 2015. Extension of the inequalities of Hardy and Hilbert, arXive: http://arxiv.org/pdf/1502.05909. pdf.

Sababheh, M. 2008a. Hardy-type inequality on the real lines. $J$. of Ineq. in Pure and Applied Math. 9(3): No. 72.

Sababheh, M. 2008b. On an argument of Korner and Hardy's inequality. Analysis Mathematica 34: 51-57.

Zhu, K. 2004. Translating inequalities between Hardy and Bergman spaces. Amer. Math. Monthly. 111(6): 520-525.

Applied Analysis Research Unit

Department of Mathematics and Statistics

Prince of Songkla University

Hatyai, Songkhla, 90110

Thailand

*Corresponding author; email: kamthorn.c@psu.ac.th

Received: 31 August 2016

Accepted: 18 January 2017 